\title{
FIXED POINT THEOREMS FOR CERTAIN CLASSES OF MULTIFUNCTIONS
}

\author{
R. E. SMITHSON
}

\begin{abstract}
The following two fixed point theorems for multifunctions are proved: THeOREM. If $X$ is a tree and if $F: X \rightarrow X$ is a lower semicontinuous multifunction such that $F(x)$ is connected for each $x \in X$, then $F$ has a fixed point. THEOREM. Let $X$ be a topologically chained, acyclic space in which every nest of topological chains is contained in a topological chain. If $F: X \rightarrow X$ is a point closed multifunction such that the image of a topological chain is chainable and such that $F^{-1}(x)$ is either closed or chainable for each $x \in X$, then $F$ has a fixed point.
\end{abstract}

1. Introduction. Numerous papers have been published which give conditions under which certain topological spaces will have the fixed point property for a class of functions. For example, [1], [2], [6], [8], and [10] all give fixed point theorems for classes of multivalued functions. However, in all of these cases the theorems are all for upper semicontinuous or continuous multifunctions. The purpose of this paper is to prove two fixed point theorems with much different hypotheses. In the first of these we use lower semicontinuity rather than upper semicontinuity. In the second we exploit the observation that the preservation of connectedness or arcwise connectedness by continuous maps is an essential ingredient in the proofs of many fixed point theorems by assuming that the function in question preserves arcwise connectedness. In so doing it is not necessary to assume any of the classical forms of continuity. This is much the same idea used in [3]. Furthermore, the present Theorem 2 implies several other known theorems which do use continuity. Among these are Ward's Theorem [10] and a theorem of G. S. Young [11]; these are given as corollaries.

The term multifunction or multivalued function denotes a correspondence $F: X \rightarrow Y$ such that $F(x)$ is a nonempty subset of $Y$ for each $x \in X$ (i.e., $F$ is a relation on $X$ into $Y$ ). If $A \subset X$, then $F(A)=\bigcup\{F(x): x \in A\}$, and if $B \subset Y$, then $F^{-1}(B)=\{x \in X: F(x) \cap B \neq \varnothing\}$ where $\varnothing$ denotes the empty set. A multifunction $F: X \rightarrow Y$ is lower semicontinuous (1.s.c.) iff for each open set $U \subset Y$, the set $F^{-1}(U)$ is an open subset of $X$. Further, $F$ is upper semicontinuous (u.s.c.) iff the inverse of each closed set is closed,

Received by the editors February 9, 1971.

AMS 1969 subject classifications. Primary 5485, 5465; Secondary 5456.

Key words and phrases. Fixed point theorems for multifunctions, tree, arcwise connected spaces, topologically chained spaces, lower semicontinuous multifunctions.

(c) American Mathematical Society 1972 
and $F$ is continuous iff it is both 1.s.c. and u.s.c. Finally, the multifunction $F$ is called point closed (connected, compact, etc.) in case $F(x)$ is closed (connected, compact, etc.) for all $x \in X$.

A useful characterization of lower semicontinuity is given in the following lemma whose proof follows directly from the definition and is omitted.

Lemma 1. A multifunction $F: X \rightarrow Y$ is l.s.c. iff for each open set $U \subset Y$ and each $x \in X$ with $F(x) \cap U \neq \varnothing$, there exists an open set $V \subset X$ such that $x \in V$ and $F(z) \cap U \neq \varnothing$ for all $z \in V$.

A fixed point of a multifunction $F$ is a point $x$ such that $x \in F(x)$.

2. The fixed point theorems. The proofs of the two fixed point theorems are modifications of order theoretic arguments used in earlier works of several authors (see, for example, [3] and [10]). For this reason we first give the necessary preliminary material and the statements of the theorems. Then we present a lemma that can be used in proving both theorems, and then we give the proofs of the main theorems.

In [6] Wallace proved that a tree had the fixed point property for upper semicontinuous multifunctions for which $F(x)$ was compact and connected for each $x \in X$. Our first theorem asserts that each tree has the fixed point property for point connected, l.s.c. multifunctions. Before stating this theorem we review the basic properties of trees with particular emphasis on their partial order structure.

Let $X$ be a continuum. We say that $z$ separates $x$ and $y$ in $X$ if and only if $X \backslash z=A \cup B$ where $A$ and $B$ are separated and $x \in A, y \in B$. Then a continuum $X$ is a tree if and only if each two distinct points of $X$ are separated by a third point in $X$.

We define a relation $\leqq$ on $X$ as follows: Let $e \in X$ be fixed. Then $x \leqq y$, $x, y \in X$, if and only if either (i) $x=e$, (ii) $x=y$ or (iii) $x$ separates $e$ and $y$. It is not difficult to show that $\leqq$ is a partial order on $X$ and that $e$ is a unique minimal element. The principal properties of this partial order are given in the following lemma which is from [7].

LEMmA 2. The partial order $\leqq$ satisfies:

(i) $\leqq$ is semicontinuous.

(ii) $\leqq$ is order dense.

(iii) If $x, y \in X$, then $L(x) \cap L(y)$ is a nonempty chain.

(iv) $M(x) \backslash x$ is open for all $x \in X$.

In Lemma $2, L(x)=\{y: y \leqq x\}$ and $M(x)=\{y: x \leqq y\}$.

Then $\leqq$ is semicontinuous if and only if $M(x)$ and $L(x)$ are closed sets for all $x \in X$. As usual $x<y$ means $x \leqq y$ and $x \neq y$. Then $\leqq$ is order dense if 
and only if $x<y$ implies there exists a $z \in X$ such that $x<z<y$. Finally, a chain is any linearly ordered subset.

It follows from the compactness of $X$ and Lemma 2 that each chain in $X$ has a supremum in $X$, and that each closed chain contains its supremum (see for example [10]).

We also need the following lemma in which we assume that $X$ is a tree with the partial order $\leqq$.

Lemma 3. If $A$ is connected, if $x \notin A$, and if $A \cap M(x) \neq \varnothing$, then $A \subset M(x)$.

Proof. Suppose $A \cap M(x) \neq \varnothing$ and $A \cap(X \backslash M(x)) \neq \varnothing$. Then $A \cap$ $\{M(x) \backslash x\}$ and $A \cap(X \backslash M(x))$ are relatively open subsets of $A$ and since $x \notin A$, these separate $A$ contrary to the assumption that $A$ is connected.

THeOrem 1. Let $X$ be a tree and let $F: X \rightarrow X$ be a point connected, l.s.c. multifunction on $X$ into $X$. Then there exists a point $x \in X$ such that $x \in F(x)$ (i.e., $F$ has a fixed point).

REMARK. In the theorems using upper semicontinuity or continuity it was generally necessary to assume that each set $F(x)$ was compact as well as connected. But in Theorem 1 we did not need to assume that these sets are compact.

For the next theorem we use the terminology and definitions from [9] which are reviewed below.

A topological chain is a continuum with exactly two noncutpoints which are called endpoints. A topological chain with endpoints $x, y$ is written $[x, y]$. In this context $\{x\}=[x, x]$ is a topological chain. If a topological chain is also separable then it is a real arc. A space (or subset of a space) is chainable if and only if each pair of points in $X$ is contained in a topological chain in $X$ and such a space is acyclic in case for each $x, y \in X$ there is only one topological chain in $X$ with endpoints $x, y$. If $X$ is an acyclic chainable space, $X$ is partially ordered as follows: Let $e \in X$ be fixed. Then $x \leqq y$ iff $[e, x] \subset[e, y]$. It is easy to verify that $\leqq$ is a partial order on $X$ and $e$ is the unique minimal element. As above we set $M(x)=\{y: x \leqq y\}$, and in the following the partial order referred to is this one.

Now if $X$ is a topologically chained space in which each nest of chains is contained in a chain, $X$ is acyclic. Furthermore, if $S$ is a nested collection of subsets of $X$ of the form $[e, x]$, then $U S \subset[e, z]$ for some $z \in X$ and hence, $\bigcup S$ has a supremum in $[e, z]$. With these facts at our disposal we are now ready to state the next theorem.

THEOREM 2. Let $X$ be a topologically chained space in which every nest of topological chains is contained in a topological chain, and let $F: X \rightarrow X$ be a point closed multifunction on $X$ into $X$ such that $F([x, y])$ is chainable 
for each $x, y \in X$. If for each $x \in X, F^{-1}(x)$ is either chainable or closed, then $F$ has a fixed point.

Lemma 4 below asserts that under the conditions of our theorems there is a maximal element $x$ such that $y \leqq x$ implies that $F(y) \subset M(y)$. Then to conclude the proofs of the theorems we shall show that if $x$ is not a fixed point, then it can not be maximal with respect to the above stated property.

Lemma 4. Let $X, F$ satisfy either the hypotheses of Theorem 1 or Theorem 2, and let $\leqq$ be the appropriate partial order defined above with minimal element e. Further, assume that $F$ does not have a fixed point. If $e \leqq y<x$ implies that $F(y) \subset M(y)$, then $F(x) \subset M(x)$. Consequently there is an element $x_{0} \in X$ which is maximal with respect to $e \leqq y \leqq x_{0}$ implies that $F(y) \subset M(y)$.

Proof. First suppose that $X$ is a tree and that $F$ is l.s.c. If $z \in F(x) \backslash M(x)$, then, since $X$ is a tree and $F$ is l.s.c., there exist open sets $U, V$ such that $z \in U, x \in V, U \cap V=\varnothing, U \cap M\left(x^{\prime}\right)=\varnothing$ for all $x^{\prime} \in V$ and $F\left(x^{\prime}\right) \cap U \neq \varnothing$ for all $x^{\prime} \in V$. But there exists a $y \in V$ such that $e \leqq y<x$, and the above implies that $F(y) \notin M(y)$. Hence, $F(x) \subset M(x)$.

Next suppose that the hypotheses of Theorem 2 holds, and that $F(x) \nsubseteq M(x)$. In this case $F(x)$ is closed, and since $[e, x]$ is a continuum, there exists a $y \in[e, x]$ such that $F(x) \cap M(y)=\varnothing$. But $F([y, x])$ is chainable, and if $y \leqq z<x, F(z) \subset M(z) \subset M(y)$. Hence, $F([y, x])$ meets $M(y)$ and $X \backslash M(y)$ and thus contains $y$. But this is impossible unless $y$ is a fixed point. Therefore we conclude that $F(x) \subset M(x)$.

Finally, the collection $\mathscr{S}=\{[e, x]: y \in[e, x]$ implies $F(y) \subset M(y)\}$ is partially ordered by inclusion, and if $\mathscr{C}$ is a nest in $\mathscr{S}$, then the first part of the lemma implies that $\mathscr{C}$ has an upper bound in $\mathscr{S}$. Thus by Zorn's Lemma, there is a maximal element $\left[e, x_{0}\right] \in \mathscr{S}$ and $x_{0}$ is the required maximal element with the given property.

We are now ready to complete the proofs of Theorems 1 and 2. In these we shall use the above notation.

Proof of Theorem 1. Suppose that $F$ does not have a fixed point and suppose that $x \in\left[e, x_{0}\right]$ implies that $F(x) \subset M(x)$. Now let $y \in F\left(x_{0}\right)$ and let $x_{0}<z<y$. Since $F$ is 1.s.c., there exists an open set $U$ containing $x_{0}$ such that $F(x) \cap(M(z) \mid z) \neq \varnothing$ for all $x \in U$. Further, we may choose $U$ so that $U \cap M(z)=\varnothing$. Now, since $X$ is a tree there is a $z_{1} \in U$ such that $x_{0}<z_{1}$ and $\left[x_{0}, z_{1}\right] \subset U$. Then, if $x_{0} \leqq x \leqq z_{1}, F(x) \cap M(x) \neq \varnothing$ and thus $F(x) \subset$ $M(x)$ (since $F$ is point connected). This implies that no such element $x_{0}$ is maximal with respect to $x \in\left[e, x_{0}\right]$ implies $F(x) \subset M(x)$. Since this contradicts Lemma 4, we conclude that $F$ has a fixed point.

Proof of Theorem. 2. Apply Lemma 4 to obtain an element $x_{0}$ which is maximal with respect to $F(x) \subset M(x)$ for all $e \leqq x \leqq x_{0}$. Then let $x_{1}$ be the 
minimal element of $F\left(x_{0}\right)$, and let $z_{1} \in\left[x_{0}, x_{1}\right]$ be such that $F\left(z_{1}\right) \notin M\left(z_{1}\right)$. (Note that $x_{0}<z \leqq x_{1}$.) Then, since $F\left(\left[x_{0}, z_{1}\right]\right)$ is arcwise connected there is an $x \in\left[x_{0}, z_{1}\right] \cap F\left(\left[x_{0}, z_{1}\right]\right)$ such that $x \neq x_{1}$. Now we have two possibilities; either $F^{-1}(x)$ is closed or $F^{-1}(x)$ is chainable. First, if $F^{-1}(x)$ is closed, then $F^{-1}(x) \cap\left[x_{0}, z_{1}\right]$ is compact and thus has an infimum, $y_{1}$, and $y_{1} \neq x_{0}$. Now let $x_{0}<y_{2}<y_{1}$. If $F(z) \subset M(z)$ for all $z \in\left[x_{0}, y_{2}\right]$ we contradict the maximality of $x_{0}$. On the other hand we have $x_{1} \in F\left(\left[x_{0}, y_{2}\right]\right)$ and for some element $z$ of $\left[x_{0}, y_{2}\right], F(z) \notin M(z)$ which implies that $x \in F\left(\left[x_{0}, y_{2}\right]\right)$ which contradicts the definition of $y_{1}$. Thus assume that $F^{-1}(x)$ is chainable, and hence $F^{-1}(x) \cap\left[x_{0}, z_{1}\right]=A$ is an arc. If inf $A \neq x_{0}$, we obtain the same contradiction as above. So suppose inf $A=x_{0}$, and let $x_{0}<y_{1}<x$. Then if $z \in\left[x_{0}, y_{1}\right], F(z) \cap M(z) \neq \varnothing$ (as $\left.x \in M(z)\right)$, which implies that either $z \in F(z)$ or $F(z) \subset M(z)$. The latter case contradicts the maximality of $x_{0}$. Hence, we conclude that $F$ has a fixed point.

As corollaries to Theorem 2 we obtain a theorem of Young [11], a theorem of Ward [10] and a theorem of Smithson [3].

Corollary 1 (Young). Let $X$ be an arcwise connected $T_{2}$-space in which each nest of arcs is contained in an arc. If $f: X \rightarrow X$ is a continuous function of $X$ into $X$, then $f$ has a fixed point.

Proof. Since $f$ is continuous, the image under $f$ of an arc is arcwise connected and $f^{-1}(x)$ is closed for each $x$. Thus Theorem 2 implies that $f$ has a fixed point.

COROLlary 2 (WARD). Let $X$ be a hereditarily unicoherent, chainable continuum. If $F: X \rightarrow X$ is an u.s.c. multifunction such that $F(x)$ is a subcontinuum for each $x \in X$, then $F$ has a fixed point.

Proof. Since $X$ is hereditarily unicoherent and chainable, each subcontinuum is chainable, and since $F$ is u.s.c., $F^{-1}(x)$ is closed for each $x \in X$. Thus Theorem 2 applies.

Corollary 3 (Smithson). Let $X$ be a tree, and let $F: X \rightarrow X$ be a point closed multifunction. If for each connected set $C \subset X$ both $F(C)$ and $F^{-1}(C)$ are connected, then $F$ has a fixed point.

Proof. Since every connected subset of a tree is chainable, $F(x)$ and $F^{-1}(x)$ is chainable for each $x \in X$, thus Theorem 2 applies.

REMARK. Observe that the space in Theorem 2 need not be compact nor $T_{2}$, nor locally connected. While it is true that under certain conditions a multifunction which preserves connected sets and has closed point inverses is u.s.c., this usually requires the domain to be locally connected. (See for example [4] and [5].) On the other hand simple examples are given in [3] which show that the preservation of arcwise connectedness by 
both $F$ and $F^{-1}$ does not imply either u.s.c. or 1.s.c. Finally note that point connected u.s.c. or l.s.c. multifunctions preserve connected sets but not arcwise connected sets and point compact u.s.c. multifunctions preserve compact sets but l.s.c., point compact multifunctions do not preserve compact sets.

Finally in 1962 in his dissertation at the University of Oregon, K. Harris showed that the continuous image of a topological chain in a $T_{2}$-space is chainable. Thus it is not necessary to assume that the arcs in Corollary 1 are separable.

\section{REFERENCES}

1. R. L. Plunkett, A fixed point theorem for continuous multi-valued transformations, Proc. Amer. Math. Soc. 7 (1956), 160-163. MR 19, 301.

2. R. E. Smithson, Changes of topology and fixed points for multivalued functions, Proc. Amer. Math. Soc. 16 (1965), 448-454. MR 30 \#5293.

3. - A fixed point theorem for connected multi-valued functions, Amer. Math. Monthly 73 (1966), 351-355. MR 33 \#1848.

4. — On criteria for continuity, Nieuw Arch. Wisk. (3) 14 (1966), 89-92. MR 33 \#6599.

5. - A note on the continuity of multifunctions, J. Natur. Sci. and Math. 7 (1967), 197-202. MR 38 \#5176.

6. A. D. Wallace, A fixed-point theorem for trees, Bull. Amer. Math. Soc. 47 (1941), 757-760. MR 3, 57.

7. L. E. Ward, Jr., A note on dendrites and trees, Proc. Amer. Math. Soc. 5 (1954), 992-994. MR 17, 180.

8. - A fixed point theorem for multi-valued functions, Pacific J. Math. 8 (1958), 921-927. MR 21 \#2215.

9. - A A fixed point theorem for chained spaces, Pacific J. Math. 9 (1959), 12731278. MR 21 \#7496.

10. - Characterization of the fixed point property for a class of set-valued mappings, Fund. Math. 50 (1961/62), 159-164. MR 24 \#A2956.

11. G. S. Young, Jr., The introduction of local connectivity by change of topology, Amer. J. Math. 68 (1946), 479-494. MR 8, 49.

Department of Mathematics, University of Wyoming, Laramie, Wyoming 82070 